\title{
La evaluación en la innovación curricular: Percepciones y prácticas de los docentes en torno a la evaluación*
}

Mara Elgue Patiño**

\section{Resumen}

Este artículo sintetiza una investigación que se propuso conocer las percepciones de los profesores de Educación Media Superior sobre la evaluación en un contexto de innovación curricular. El nuevo sistema propone evolucionar de un modelo finalista centrado en el examen hacia una evaluación de procesos.

La evaluación es uno de los procesos que reviste mayor interés en los cambios. Implica corregir y transformar las tareas de gestionar, enseñar y aprender. Cualquier cambio curricular, está íntimamente vinculado con el profesor en tanto hacedor del currículo en acción. Por ello importan sus opiniones y creencias implícitas para ver lo que efectivamente hacen a la hora de evaluar.

Entre las principales conclusiones, se señala que las prácticas de evaluación centradas en la función social de la acreditación han primado en detrimento de la función reorientadora de los aprendizajes y de las prácticas de enseñanza.

\begin{abstract}
This article summarizes the research made in order to know Secondary School Education teachers' opinion in an innovative curricular context.

Evaluation in one of the processes that involves more interest in changes.

It implies correcting and transforming the tasks of administrating, teaching and learning. Any curricula change is deeply related to the teacher as long as the teacher is building an ongoing curricula.

Among its main conclusions, it's clearly shown that those assessment practices centered on the social function of certificating qualifications have been ontstanding at the expense of the reorientating function of learning and teaching practices.
\end{abstract}

\section{I ntroducción}

La Administración Nacional de Educación Pública del Uruguay (ANEP), ha puesto en marcha innovaciones curriculares a partir de planes educativos experimentales para la educación media superior o bachillerato que constituyen una respuesta a nuevas realidades sociales. Ante un crecimiento sostenido de la matrícula, la educación pública, logró una alta cobertura en el bachillerato; sin embargo, esta no ha sido acompañada por un incremento significativo de egresos, debido a altas tasas de fracaso, repetición, rezago y deserción. ${ }^{1}$

El diagnóstico de la educación media superior, arroja datos alarmantes referidos al desgranamiento de la matrícula, si se tiene en cuenta que de cada 100 estudiantes que inician el bachillerato sólo 30 lo culminan y de estos 30 sólo 10 lo hacen en los plazos previstos. Estos hechos condujeron a las autoridades de la enseñanza pública a diseñar planes de estudio que apuntaron a cambios sustanciales, entre los que el sistema de evaluación ocupó un lugar de privilegio. 
La pregunta que guía el presente trabajo es cómo perciben los docentes de un Liceo de Bachillerato del suroeste de Colonia, la evaluación del Plan experimental TEMS ( Transformación de la Educación Media Superior) ${ }^{2}$ y cómo la implementan en sus prácticas educativas. Este cambio curricular propone desarticular una cultura de evaluación centrada en el examen final, presente en el plan anterior, entre cuyos efectos no deseados se registró un alto nivel de rezago para dar lugar a un sistema de evaluación que permita valorar los procesos a partir de instancias de corte como pruebas semestrales y proyectos interdisciplinarios. ${ }^{3}$

La investigación se propuso describir, analizar e interpretar cómo se articula un nuevo sistema de evaluación desde el currículo prescrito al currículo en acción, observando los procesos de recepción e implementación de las innovaciones curriculares llevadas a cabo. Partimos de la base de que la evaluación educativa, además de la evaluación de aprendizajes, supone la de los procesos de enseñanza, la evaluación curricular, institucional y de políticas educativas.

Las creencias que los docentes tienen acerca de qué es enseñar y qué es aprender redundará indefectiblemente en sus propuestas áulicas de evaluación.

En la medida que las propuestas de innovación se sustenten en un diálogo con las creencias e intenciones de los docentes, probablemente mayor será la chance de que las prácticas de evaluación de corte tradicional, repetitivas y clasificadoras cedan su espacio a prácticas alternativas, comunicativas, formadoras y negociadas.

Los datos se relevaron en el Liceo $\mathrm{N}^{0} 2$ de Carmelo, departamento de Colonia, Uruguay y la unidad de observación festuvo integrada fundamentalmente por los docentes vinculados a los $1^{\text {eros }}$ de la EMS (Educación Media Superior) y entre estos, aquellos que trabajaron durante el 2003, en el plan experimental. Esta decisión se funda en que sólo estos docentes pueden, mediante una mirada retrospectiva, hacer una valoración de la nueva propuesta curricular. Se trata, de abordaje cualitativo, de carácter descriptivo, analítico e interpretativo que intenta aportar nuevas categorías de conocimiento.

El trabajo se enmarca en el estudio de caso en tanto procura ver el impacto de una innovación curricular en las prácticas de evaluación.

Dentro de las técnicas pertinentes al paradigma cualitativo se llevaron a cabo:

- Entrevistas profesionales de investigación con una pauta semiestructurada dirigidas a profesores de $1^{\text {er }}$ año de bachillerato que voluntariamente accedieron a brindar la información requerida.

- Análisis de documentos. Se estudiaron las registros producidos en el libro del profesor y las propuestas de evaluación : consignas de trabajos escritos, pruebas, informe interdisciplinario , actas de salas generales y salas de asignatura.

- Observación- Esta técnica está fundada en la necesidad de registrar no sólo la evaluación en tanto producto palpable en un documento, sino a partir de los procesos que se gestan en el aula, en las salas docentes o en el ámbito de las comisiones de trabajo.

\section{La indagación se llevó a cabo en tres niveles:}

- Evaluación del currículo y su implementación en el marco de la Institución liceal.

- Evaluación de los aprendizajes.

· Evaluación de la enseñanza. 
Se fundamenta la indagatoria, en un escenario donde comienzan a escucharse voces de malestar o desconcierto. Entre los docentes algunos sostienen que es necesario hacer a un lado la pedagogía de la respuesta, pero es probable que no se cuente con necesaria pericia profesional ni con una cultura institucional que facilite los procesos pertinentes.

Otros profesores rechazan la nueva propuesta o adoptan una postura prudentemente moderada, pues ya han visto ir y venir muchas innovaciones en las que centraron grandes expectativas y que luego fracasaron.

La desconfianza frente a las modas que prometen sustanciales mejoras educativas, los impulsa a defender las prácticas tradicionales que estiman han probado su eficiencia, y frente a las cuales se sienten seguros y confiados.

\section{Algunos aportes desde la teoría}

Teniendo en cuenta el alto índice de deserción y fracaso registrado a nivel del bachillerato, según reciente estudio llevado a cabo por la Comisión TEMS ${ }^{4}$, la misma, concluye afirmando que el Bachillerato demanda una inminente revisión.

La síntesis de la propuesta curricular que responde a la demanda de revisión, señala que se desea generar un cambio que impacte en las prácticas educativas permitiendo eliminar: el enciclopedismo, los planteos rutinarios y memorísticos, la información desjerarquizada, sin pertenencia ni utilidad, así como la evaluación basada en la mera evocación memorística de conocimientos, desconectada, por ende, del aprendizaje.

Entre las finalidades de este proyecto se destaca: "La formación de los educandos para la vida, la ciudadanía, el trabajo y la prosecución de estudios a nivel superior. Para ello se introduce una propuesta de formación por competencias que son entendidas como conocimiento en acción y que se alcanzarán sólo a través de contenidos"

Entre las innovaciones que presenta el currículo se destacan:

- Un régimen de evaluación que aspira a obtener información sobre los procesos pedagógicos que se dan en la institución educativa, analizando e interpretando los mismos para su reorientación. Los componentes de la evaluación son: valoración del proceso, pruebas semestrales y un trabajo interdisciplinario anual. Se introducen mecanismos de recuperación y compensación de insuficiencias académicas con suficiencias en otras asignaturas que integren el mismo trayecto, siempre que el promedio de calificaciones del trayecto alcance la nota mínima exigida. (58 en una escala de 1 a 100)

- Actividades descentralizadas. Cada Centro de acuerdo con su disponibilidad de recursos humanos y materiales y teniendo especialmente en cuenta los intereses de los estudiantes realizará una oferta de actividades descentralizadas optativas, que tiendan al desarrollo integral y a brindar apoyo al estudiante.

- Salas docentes, salas de trayecto y salas de asignatura que constituyan espacios organizacionales extra-áulicos de coordinación, discusión y reflexión.

En lo referido al perfil de egreso del estudiante de educación media superior, la Propuesta Curricular señala entre sus puntos medulares: -el dominio de competencias comunicativas que posibiliten la adquisición de conocimientos y sentimientos. -el desarrollo de competencias necesarias tanto para acceder a estudios terciarios como para incorporarse al mundo del trabajo y el desarrollo del pensamiento crítico, la autonomía intelectual y la formación ética. 


\section{La cultura del examen y del escrito.}

Díaz Barriga (1992), realiza una interesante síntesis de la evolución de los sistemas de evaluación desde la antigüedad pasando por la edad Media hasta nuestros días. Plantea la hipótesis de que el examen, se constituye como un espacio de inversión de relaciones, en tanto convierte los problemas sociales en pedagógicos; los metodológicos en problemas de examen y los problemas teóricos de la educación quedan circunscritos al ámbito técnico de la evaluación. Ahondando en estos aspectos, destacamos las siguientes funciones del examen:

1. Legitima el saber de una persona, acreditándolo, permitiendo o denegando su ingreso y permanencia, a un determinado sistema. Emparentándose con los postulados de las ciencias experimentales, el centro de interés se sitúa en la eficacia de la acción. Los problemas de orden social, posibilidad de acceso y permanencia en los centros educativos, justicia social etc, se trasladan a problemas de orden técnico: objetividad, validez y confiabilidad del instrumento, que no puede ser justo cuando la estructura social es injusta. Bajo la apariencia de objetividad, el examen se transforma en un instrumento de restricción del acceso a la educación, mediante justificaciones académicas constituyéndose en un auténtico fetiche pedagógico.

2. Invierte los problemas metodológicos en problemas de rendimiento. Ya se mencionó que el examen ingresa al escenario educativo en la Edad Media, aunque inicialmente sólo se usaba como un espacio público para mostrar la competencia que se había adquirido. En la Didáctica Magna Comenio lo vincula indisolublemente al método: cuando el alumno no aprende el maestro debe revisar el método.

En síntesis el examen se convirtió en un espacio independiente del ámbito áulico y más ampliamente en un segmento añadido al currículo y no en una parte integral del mismo.

La función de acreditación apartó el placer del joven por saber y el mismo fue sustituido por la preocupación de salvar el examen. Las formas y contenidos del examen se apartaron de los intereses de los estudiantes y descuidaron notoriamente los problemas de la formación y del aprendizaje. En forma genérica podemos decir que el currículo en su conjunto se alejó del conocimiento útil y práctico.

Santos Guerra (1996) plantea algunas consideraciones teóricas a tener presentes a la hora de evaluar. Establece la distinción entre evaluación sumativa y formativa. La primera está centrada en el logro de ciertos objetivos finales y la actividad que suscita más atención, es el estudio de cómo mejorar la forma de conseguir lo propuesto. La evaluación formativa, por su parte, supone la atención de los procesos; está integrada al currículo, lo desarrolla y perfecciona ya que posibilita información para la reorientación y toma de decisiones.

Si entendemos la evaluación como una simple revisión de productos finales, que centra la mirada en los resultados académicos de los estudiantes, perdemos la consideración de la escuela como ecosistema, en el que confluyen un entramado de interrelaciones que cobran sentido en su propio contexto externo ya que la escuela está inserta en una comunidad y forma parte de un sistema educativo. Por otra parte, hay un contexto interno que supone redes comunicativas entre estudiantes y sus culturas familiares, profesores, directores entre quienes se comparte una cultura de centro con sus normas explícitas e implícitas y un conjunto de acciones o cosas que suceden en la escuela y sus respectivos códigos de interpretación.

El proceso de cambio hacia una evaluación sistémica y formativa, supone hacer operativos los espacios organizacionales de meta -análisis, salas docentes, jornadas de coordinación y 
actualización; hacer un uso eficiente de los instrumentos de evaluación pruebas, informe interdisciplinario, trabajos áulicos y de los nexos con los estudiantes, a partir de la interrelación con el adscripto tutor así como la implementación de los procesos de autoseguimiento.

El examen ocupó un lugar preponderante en la cultura escolar, en tanto la importancia que reviste en su función de control y clasificación de los estudiantes en función de las calificaciones.

Como motivación extrínseca, impuesta desde afuera, el examen conduce a la repetición de contenidos y a la pasividad. Los estudiantes concentran sus esfuerzos en el examen. La motivación intrínseca se halla ausente. Como consecuencia, los contenidos aprendidos para los exámenes caen en el olvido rápidamente pues dejó de existir la motivación para retenerlos y los procesos sucesivos, rara vez permiten un empleo útil de los insumos que formaron parte del temario de un determinado curso. En niveles posteriores, son frecuentes las exclusiones de contenidos, tanto por parte de profesores como de estudiantes, por considerarlos pertenecientes al curso anterior, limitando la posibilidad de construir un currículo integrado, aun dentro de la misma asignatura.

Algunas sinrazones: Acreditar sin aprender, aprender sin acreditar

De lo anteriormente expuesto, se comprende el peso que la evaluación centrada en los resultados ha tenido hasta el presente en el bachillerato de la educación pública en el Uruguay.

Por una parte, los planes educativos centrados en el examen final que define la actuación del estudiante, en forma independiente de los aprendizajes que efectivamente logró a lo largo del curso, ha llevado a que los profesores, sientan con fuerza, la presión social de rendir cuentas de su trabajo a partir de los resultados académicos alcanzados por sus estudiantes en las instancias de examen y, simultáneamente, a vivenciar su rol de evaluador como una fuente de poder.

Bajo estas normas, es previsible que el docente trabaje para el examen, todo su accionar está orientado hacia el horizonte del examen final, motivado por la responsabilidad de que sus estudiantes aprueben.

La respuesta estudiantil, por su parte, es la pasividad, ya que finalmente si lo que cuenta es el examen, pocos están dispuestos a colectivizar sus inquietudes y dudas; en última instancia el examen es individual y por tanto es problema de cada quien ver cómo mejor se sortean las dificultades para llegar a un resultado satisfactorio. Los cursos paralelos, individuales, a cargo de profesores particulares son una consecuencia previsible y la característica de los mismos está centrada exclusivamente en descubrir y dominar la mecánica del examen.

En las aulas, la evaluación pone la mira en el estudiante. Los escritos mensuales se convierten en aprestamientos para la prueba final y tienen un peso notoriamente superior al de otros instrumentos de evaluación, a la hora de poner las notas en el libro del profesor. El mito del escrito ensombrece cualquier otra producción del estudiante, la cual independientemente de su valor en tanto creatividad o nivel reflexivo, caerá en el mejor de los casos como una nota más en el casillero de los "orales".

Todas la previsiones que el estudiante logre hacer, acerca de las preferencias bibliográficas y temáticas de sus profesores, así como su habilidad para sacar notas mientras éstos exponen, registrando máximas, definiciones y conceptos valorados como importantes, contribuirán sustancialmente a edificar su oficio de alumno, aunque esto no implique procesos de aprendizaje genuinos. De manera que cada asignatura se convierte en un objeto más de consumo: se acopian los materiales, se repiten y se olvidan una vez aprobado el examen. 
Obviamente, esta generalización simplifica en extremo una realidad que felizmente es bastante más compleja. Si bien todos tenemos algún par de asignaturas aprobadas en el bachillerato sin que podamos decir de ellas mucho más que el objeto de estudio, lo cierto es que a pesar de estas prácticas enciclopedistas, construimos nuestro capital cultural con conocimientos útiles, saberes de los que ningún examen se ocupó, pero que cambiaron nuestra interacción con el mundo circundante, incluyendo saberes adquiridos aún en cursos no aprobados.

En síntesis, el sistema de evaluación privilegia la función social de acreditación, la cual prevalece notoriamente en detrimento de la función reorientadora de los aprendizajes y de las prácticas de enseñanza. La evaluación termina clasificando estudiantes según su éxito o fracaso sin que esto guarde una necesaria correlación con los aprendizajes, de manera tal que se puede culminar el ciclo acreditando sin aprender o con un desarrollo muy limitado de competencias y capacidades.

\section{Modelos de evaluación}

La concepción de la evaluación, de una acentuada polisemia ha mostrado variaciones en el tiempo. J. Manuel Álvarez Méndez distingue dos perspectivas históricas: la cuantitativa y la cualitativa. El autor aclara que el término evaluación ha compartido el espacio semántico con los términos medición y calificación. Propone un deslinde terminológico.

La vinculación de la evaluación con la medición vino de la mano de la investigación experimental próxima al campo de la psicología, siendo la psicometría su referente más directo. La evaluación según el modelo experimental consiste en medir resultados.

La Pedagogía por objetivos contribuyó a reforzar la asociación medición = evaluación, en tanto puso especial interés por las pruebas objetivas de tipo test. Desde esta perspectiva, evaluar implica comparar el aprendizaje de entrada con el resultado final de salida o producto observable en la conducta manifiesta del estudiante. Según este paradigma las evaluación es una técnica que se operativiza como el segmento final de un proceso y que se acerca al concepto de medición y de calificación en tanto, la conducta, toma concreción en una asignación numérica correspondiente a una escala de notas.

Parte de la base de que los fines educativos consisten en operar cambios en los seres humanos. Si los cambios deseados, se traducen en el cumplimiento de objetivos que puedan plasmarse en conductas constatables, la evaluación consistirá precisamente, en verificar los resultados del aprendizaje en términos de productos finales, alcanzados o no.

La evaluación centrada en objetivos deja escaso margen de participación al sujeto evaluado, que no define y en ocasiones ni siquiera conoce los objetivos de las actividades educativas que desarrolla. Por tanto no hay forma de lograr autonomía cognitiva ni de acción. Sin duda, es un enfoque restrictivo, en tanto aprende quien logra la conducta esperada y explícita en los objetivos y quien responde de otra manera fracasa.

Un sinnúmero de aprendizajes quedan al margen, sin valoración, ni calificación, sencillamente por no estar contemplados en los objetivos. Por otra parte, la aparente objetividad de un aprendizaje, constatado a partir de una respuesta exitosa no es tal, ya que conductas memorísticas y repetitivas pueden recibir la aprobación, sin que tengan lugar los procesos cognitivos que caracterizan al aprendizaje.

Finalmente el modelo de evaluación basado en objetivos operativos, al poner el énfasis en forma desmedida en los resultados finales, genera la obsesión por el examen desvirtuando el proceso educativo ya que la mayor preocupación del estudiante es aprobar, en detrimento del proceso de aprendizaje. 
La evaluación desde la perspectiva cualitativa supone la descripción e interpretación de los distintos momentos del proceso educativo. El referido autor aclara que toda evaluación es cualitativa, en tanto evaluar significa precisamente, valorar, justipreciar e interpretar los datos obtenidos. El profesor evaluador instalado en una actitud cualitativa, debe redefinir su propia función y orientarla más hacia la investigación, la coparticipación, deliberación e innovación.

La metodología cualitativa, exige tomar conciencia de cada decisión a partir del análisis e interpretación del proceso educativo. Parte de la base de que si el aprendizaje es una construcción, solo el constructor puede decir lo que construye y por lo tanto, el sujeto evaluado participa activamente en el proceso evaluador. Se genera de esta forma una suerte de triangulación, ya que al menos se cuenta con dos fuentes de información (alumno y profesor) a las que en ocasiones se suma un evaluador externo, enriqueciendo la información.

Es un modelo dinámico, porque los sujetos inmersos en el proceso de aprender, realizan un seguimiento estrecho de las actividades educativas día a día.

Sintetizando, podemos distinguir tres modelos:

- Evaluación experimental. Su función primordial es medir y controlar resultados con un instrumento estandarizado, que permita arribar a datos numéricos a los que se somete a un análisis estadístico. Los conceptos de validez, fiabilidad, generalización e inferencia son claves desde esta perspectiva.

- La evaluación en la pedagogía por objetivo. Este modelo entronca con la teoría conductista. Manifiesta aprendizaje quien al final del proceso instructivo cumple con los objetivos, a través de una conducta esperada y verificable.

- La evaluación cualitativa: Es sistémica y procesual en tanto tiene en cuenta los contextos amplios en los que se dan los hechos a evaluar. Centra el interés en la descripción e interpretación de lo que se está evaluando. Genera espacios para los procesos de autoevaluación y de coevaluación ya que la actividad, es compartida por los actores implicados, involucrados en un aprendizaje cooperativo y solidario tendiente a una creciente autonomía.

Careaga, A. (2003) propone una tipología binaria: Evaluación tradicional -Evaluación alternativa. La primera responde a una racionalidad técnica y estratégica mientras que la segunda responde a una racionalidad práctica y de acción comunicativa Partimos del supuesto de que existe una relación significativa entre las teorías y la acción. Por tanto, las creencias de los profesores respecto a la evaluación podrán estar en consonancia con la propuesta de transformación generando adhesión, o rechazo si dicha propuesta no contempla sus intenciones.

Probablemente quienes manifiesten preferentemente adhesión hacia la innovación, procurarán alinear sus propuestas de evaluación hacia formas alternativas próximas a la evaluación formadora, interpretativa y comunicativa, mientras que quienes manifiesten predominantemente rechazo innovar tenderán a llevar a cabo prácticas de evaluación de corte tradicional, basadas en objetivos.

\section{El siguiente mapa representa la proposición expuesta:}

1 El bachillerato comprende los cursos de $4^{\circ}, 5^{\circ}$ y $6^{\circ}$ de la educación media también identificados como $1^{\circ}, 2^{\circ}$ y $3^{\circ}$ de la Educación Media Superior (EMS) . Los planes experimentales a los que se hace referencia fueron el 1993, el 2003 y la reformulación 2006. Todos tienen en común la aspiración de lograr una mayor retención y mejores niveles de aprobación a partir de estrategias entre las que se destacan importantes cambios en el sistema de evaluación del Plan 1976. En este plan cada curso se acreditaba con un examen final, de manera que la 
actuación del estudiante durante el año tenía poca relevancia en la acreditación, lo que condujo a una actitud caracterizada por la pasividad del estudiante cuya preocupación giró en torno a la preparación de examen.

2 Al Plan experimental 2003 o TEMS ( Transformación de la Educación Media Superior ), le siguió el Plan Reformulación 2006 diseñado sobre la matriz del Plan 1993, conocido como Microexperiencia, aunque retoma elementos del 2003. En la actualidad ambos planes coexisten. El 2003 se mantiene en 21 Centros, con la intención de que pueda evaluarse y el 2006 se extendió en todos los bachilleratos que tenían el Plan 1976.

3 El Plan anterior es el 1976 cuyo diseño retoma elementos del Plan 1941, con orientaciones propedéuticas a las carreras universitarias de más larga tradición en nuestro país.

4 Extraído del Documento Oficial Plan TEMS 2003.

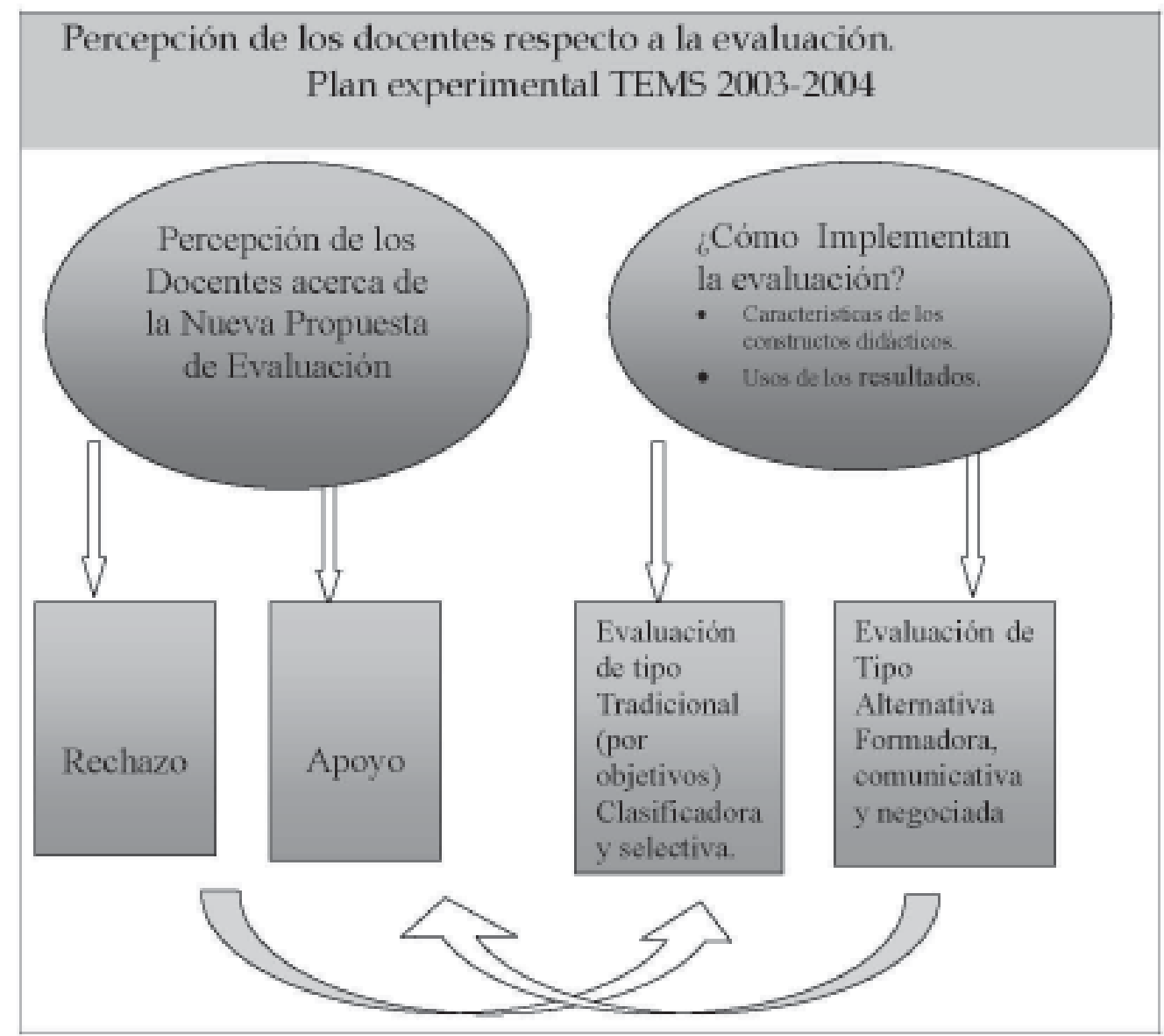


Sin duda, el intento de esquematización simplifica procesos que en la realidad se presentan con un mayor grado de complejidad, pero permite visualizar la distancia que media entre el currículo oficial y la concreción y desarrollo que tiene a partir de la mediación de las escuelas y los enseñantes. Las propuestas de descentralización del currículo al decir de Contreras, J. (1997) no se han limitado a que efectivamente los docentes interpreten y adapten el currículo, sino que también (...) ha partido de la convicción de que las reformas sólo pueden sostenerse si quienes deben realizarlas las asumen como propias.

En otras palabras, la diversificación del rol docente, comprende la responsabilidad de la adaptación y desarrollo del currículo, atendiendo a que se forma parte de una sociedad plural. El currículo requiere interpretación para su concreción, conforme a las circunstancias específicas de enseñanza, y a las necesidades particulares, aspiraciones e intereses, es decir, desde una concepción del currículo para la diversidad.

Eisner,E. (2002) al referirse al currículo operativo, diferenciándolo del previsto, sostiene que ningún currículo puede ser seguido como si fuera un guión, ni puede enseñar por sí mismo, de manera que la forma de interpretarlo y operativizarlo es crucial, y de ahí la importancia que se otorga a la dimensión pedagógica en los cambios curriculares.

Por otra parte las creencias de los docentes, están evidentemente sesgadas por el tiempo histórico que les toca vivir; desde esta perspectiva Liston y Zeichner (1993) realizan un interesante planteo referente al impacto de las tradiciones, en tanto argumentos que se mantienen a lo largo del tiempo en la formación del profesorado.

El peso de la tradición, en el pensamiento relativo a qué es enseñar y aprender, define de manera importante el rol del profesor. Dichos autores procuran identificar las tradiciones educativas significativas y ver la relación existente entre las creencias de los profesores referidas a la educación, las grandes tradiciones del pensamiento y las prácticas educativas.

\section{Las Instituciones educativas frente a los procesos de cambio}

Numerosos autores, Santos Guerra (1996), Fullan y Hargreaves (1996), Eisner (2002), entre otros, abordan la problemática que enfrentan las instituciones que pretenden introducir cambios y destacan factores intervinientes en estos procesos, los cuales tendrán mayor o menor gravitación, conforme a las situaciones históricas, sociales y contextuales de cada institución en particular. No obstante se procura sistematizar algunas de las situaciones más recurrentes en los centros educativos que se enfrentan a procesos de cambio.

- La sobrecarga y escasez de tiempo disponible. Para realizar una evaluación consistente de seguimiento de los procesos que tienen lugar en el aula, se requiere de tiempos para el análisis, el intercambio y la devolución entre otras actividades. Los docentes están peligrosamente sobrecargados de tareas y aunque resulte paradójico la innovación es una fuente más de estrés ya que en torno a ella se multiplican las expectativas y las actividades de planificación e implementación.

- El individualismo y el aislamiento. La nueva propuesta de evaluación exige una apertura a la discusión tanto como a la participación en lo que otros profesores realizan. El docente que hasta ahora trabajaba en solitario, con pleno dominio de su aula, conforme al aislamiento físico que impone la tradicional estructura a la que Fullan y Hargreaves (1996) Ilamaron la huevera escolar, se enfrenta al desafío de abrirse a la visión ajena, y de construir una cultura de trabajo en la que unos aprendan de los otros y mejoren su profesionalismo como comunidad 


\section{Entre las prácticas repetitivas, lo innovador y lo trivial.}

Fullan y Hargreaves (1996) reconocen la importancia de los docentes participativos como fuerzas impulsoras del cambio, aunque advierten que son pocos los que están preparados para ello, por la sobrecarga de tareas, el aislamiento y la falta de movilidad del rol, entre otros factores adversos.

A las problemáticas antes referidas, concernientes a la participación activa de los docentes en la innovación, debe agregarse el problema particular de los ciclos de la carrera de los profesores (Huberman, 1991) quien a partir de sus investigaciones, sostiene que los docentes que se encuentran en la etapa final o media de la carrera, difícilmente toman la innovación con entusiasmo, no estando dispuestos a abandonar las prácticas educativas que han ejercido con competencia durante décadas.

La mejor manera de que una innovación no se produzca, es negar su existencia. Considerar que la propuesta curricular no agrega nada importante al currículo en acción y que los planteos no hacen sino confirmar lo que ya se viene haciendo, probablemente genere inmovilidad, resistencia pacífica y enlentecimiento de los procesos.

Uno de los cuestionamientos reiterados que se alzan frente a la nueva propuesta de evaluación, es el temor de abandonar los instrumentos de rutina, por constructos didácticos, escritos, trabajos interdisciplinarios que resulten triviales.

Parece probable que los profesores se encuentren mejor dispuestos a aceptar un trabajo individual escrito, extenso y repetitivo que otras formas de evaluación : portafolios, trabajos de taller, proyectos áulicos, que pretenden ser creativos suponen un mayor grado de autonomía, y muchas veces un aprendizaje colectivo. A propósito de este último punto, si bien rara vez se pone en tela de juicio las virtudes de los aprendizajes alcanzados en los subgrupos de trabajo, pocas veces son considerados como adquisiciones relevantes al momento de construir la calificación del alumno. La argumentación más recurrente es que si bien importan los logros alcanzados en los equipos de trabajo, solo el escrito, como producción individual, permite constatar los aprendizajes del estudiante.

Carbonell (2001), advierte acerca de la paradoja del doble currículo. Señala que la escuela posee sus ritos a la hora de organizar y aplicar el proceso de enseñanza. Las prácticas innovadoras suelen quedar relegadas al núcleo "light" donde se experimentan metodologías activas, unidades globalizadas, trabajos que dejan un amplio espacio a la autonomía del estudiante pero, sin embargo, a la hora de evaluar se retrocede a prácticas repetitivas de memorización. Otra dicotomía se observa entre profesores que distribuyen su tiempo en dos mitades asimétricas. La primera la organizan según sus creencias y convicciones; las segunda la ejecutan atendiendo al currículo prescrito, más por obligación moral, que por convicción.

También el alumnado percibe estas dicotomías. Las propuestas de pruebas realizadas por los profesores que procuran innovar, incluyen situaciones problemáticas y vínculos interdisciplinarios que inicialmente desconciertan a los alumnos, en tanto la cultura institucional no posee la suficiente historia en la realización de trabajos en esta línea, por lo cual no debería sorprender a los profesores que los productos iniciales frente a propuestas innovadoras, sean modestos o francamente pobres, si se pone la mira en el producto final.

Respecto del impacto de las innovaciones en las teorías del profesor, Elliot (1998) hace un valioso aporte en la línea de la investigación- acción que revela la importancia de la participación docente en forma conjunta con el experto en los procesos meta-reflexivos. Plantea poner en marcha procedimientos basados en la participación activa y democrática, contribuyendo a que 
los docentes puedan soportar el sentimiento de pérdida de autoestima que implica revisar las propias prácticas educativas.

El sentimiento de responsabilidad compartida generado en las salas docentes, podría a su vez, contribuir significativamente a disminuir dicha pérdida de autoestima, al permitir a los profesores que se percaten de que tienen problemas comunes y propicia os procesos metaanalíticos indispensables para procesar los cambios. Pensamos que es más probable que los educadores se desarrollen y prosperen en escuelas que valoren y apoyen el criterio y el saber de todos sus docentes en la búsqueda común de la mejora.

Es muy factible, que las escuelas que hagan prosperar la cultura de autoevaluación institucional y los procesos de autoseguimiento liderados por los docentes, mejoren sus chances de efectuar los cambios fundamentales.

La existencia de un autoseguimiento que recoja información sobre la opinión de alumnos, profesores y padres respecto a segmentos significativos de la implementación del nuevo currículo, jerarquizará la mirada desde dentro, de manera que las evaluaciones externas resultarán menos amenazantes.

Algunas corrientes de pensamiento advierten acerca de los riesgos de una progresiva infantilización del proceso de aprendizaje y consecuentemente de su evaluación. Predomina la falsa certeza de que es posible aprender en el vacío, aprender sin memorizar, aprender sin esfuerzo, aprender sin trabajar, aprender sin registrar. Absurdo pedagógico que hoy suele condensarse en el estribillo aprender a aprender. (Núñez, V. 2003). Se abre así el cuestionamiento referente a si los adultos han renunciado al ejercicio de la función civilizadora, en tanto la evaluación se aparta del terreno de la adquisición de los bienes culturales, la elaboración de saberes, de la construcción de conocimientos.

En otras palabras si bien es sano y esperable que los profesores rehuyan de una enseñanza sin sorpresas, de contenidos enlatados, desprovistos de atractivo y sin incógnitas, tampoco es posible hablar de educación sin hacer referencia a bienes culturales, pues se cae en el riesgo de girar en un vacío que se llene con entretenimientos y trivialidades.

Este vacío que, paradójicamente muchas veces, nace de esfuerzos democratizadores, encierra el riesgo de la masificación de la enseñanza, si no se atiende a la distribución de los contenidos de la cultura, destinando el entretenimiento para las masas y las exquisiteces de la ciencia y el pensamiento (...) para las nuevas elites (Núñez,V 2003). A la luz de este aporte teórico es comprensible, la mirada prudente o francamente desconfiada de los profesores ante los clisés de las innovaciones del sistema de evaluación bajo el rótulo de la evaluación en competencias por lo cual importa dar voz a las opiniones divergentes.

\section{En busca de la buena enseñanza}

Entre las antinomias de lo repetitivo y lo innovador se impone una reflexión en torno a la buena enseñanza. Fenstermacher (1987) señala la existencia de una dependencia ontológica entre los procesos de enseñar y aprender, pero advierte que estos no están unidos por una relación causal. Este concepto ha sesgado, históricamente, gran parte del quehacer de los educadores quienes pretendieron inferir la calidad de la enseñanza midiendo los resultados de los aprendizajes siguiendo el modelo proceso/producto. 
El aprendizaje es tarea del estudiante a través de un proceso cognitivo, que él mismo pone en marcha, movido por el deseo de aprender. La enseñanza consiste en apoyar dicho deseo de "estudiantar" (ser estudiante) y permitir la acción de estudiar.

El paradigma de la enseñanza que prevaleció en nuestras escuelas por largos años fue: enseñar-aprender-aplicar (Torp,I y Sage.S. ,1999) partiendo de conceptos que debían repetirse, para luego ser aplicados, aun sin comprender su utilidad o sin lograr establecer vínculos entre los saberes escolares y la cotidianeidad. El resultado previsible ha sido el conocimiento frágil, que cae rápidamente pues se repite para aprobar y, finalmente, olvidar. En esta concepción de la enseñanza, el profesor trae al aula saberes pertenecientes a una realidad que existe y está fuera de la vida del estudiante, quien no posee sobre ella ninguna ingerencia.

Fenstermacher (1989) sostiene que educar a un ser humano consiste en proporcionarle los medios para estructurar sus propias experiencias, de manera que la responsabilidad del enseñante se desplaza de ser un proveedor de contenidos a ser proveedor de medios para estructurar la experiencia.

\section{Categorías Resultantes}

El siguiente esquema sintetiza las categorías resultantes en los tres niveles de indagación:

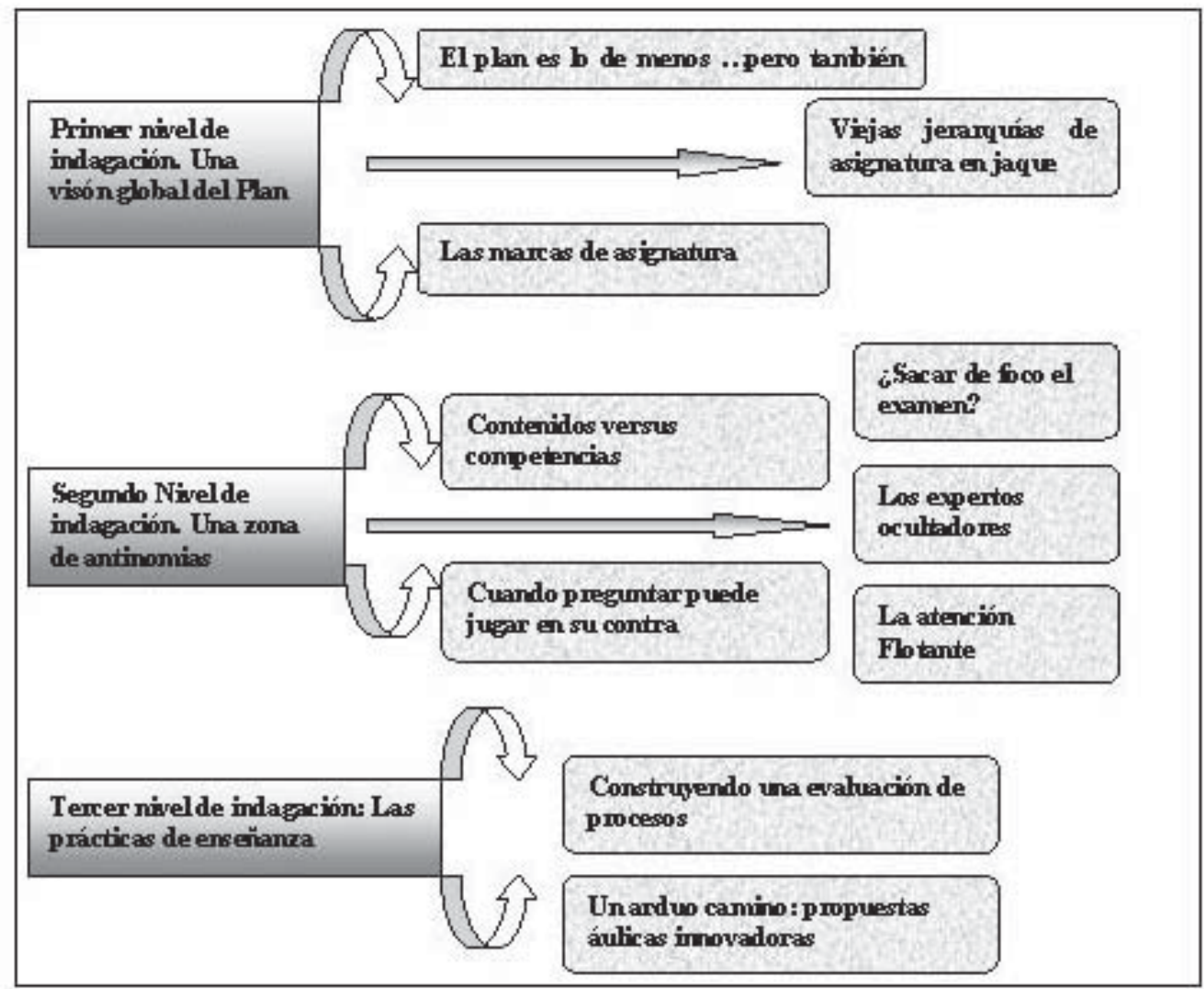

116 - Universidad ORT Uruguay 


\section{Primer nivel de indagación. Una visión global del Plan}

\section{El plan es lo de menos ... pero también importa.}

Una de las percepciones más recurrentes en los datos obtenidos es la relacionada con la visión global del Plan en cuanto a su relevancia o injerencia en las prácticas docentes. Del trabajo analítico aflora con fuerza la dicotomía entre quienes creen que un diseño curricular en consonancia con sus propias creencias, acerca de las prácticas de enseñanza, es muy relevante para potenciar y facilitar el desarrollo profesional y otro grupo de docentes que sostiene que los cambios curriculares son poco relevantes y que finalmente, no hacen más que legitimar, lo que de alguna manera ya se venía haciendo en las aulas.

\section{Viejas jerarquías de asignatura en jaque.}

Se evidenció que la innovación sitúa a todos los docentes, expertos y novatos en un plano de igualdad, barriendo con las viejas jerarquías de profesores de larga trayectoria que impusieron una modalidad finalista de evaluación .La misma, en ocasiones, produjo un efecto aberrante sobre el alumno. Una práctica muy recurrente del plan anterior ${ }^{1}$ fue promediar trimestres para obtener la calificación final del curso, desconociendo los proceso evolutivos, lo cual condujo a muchos alumnos al fracaso. Si a un estudiante se le asignaba una calificación muy baja en el primer trimestre, su evolución y esfuerzos posteriores, podían resultar inútiles en el momento de promediarse los trimestres, de manera que fueron frecuentes los abandonos a los cursos. Estas modalidades fosilizadas por la repetición de las generaciones docentes se impusieron por años.

En el nuevo currículo el sistema de evaluación, comienza siendo apenas un documento, frente una realidad educativa en la que todo está por hacerse y que requiere de las voces de expertos y novatos para su concreción. La implementación del nuevo sistema de evaluación apela al diálogo y a la negociación en un plano de igualdad que habilita el cuestionamiento de las antiguas prácticas y da lugar a las voces de todos los actores implicados.

En lo referente al diseño programático de cada asignatura, se evidenció que las estructuras muy abiertas de algunos programas, a la vez que facilitaron la contextualización, incrementaron el nivel de inseguridad frente a la selección de contenidos.

\section{Las marcas de asignatura}

La creación de espacios organizacionales, como las salas docentes semanales fueron consideradas fortalezas muy significativas para el desarrollo profesional, a la vez que instalaron un sentimiento de responsabilidad colectiva y de misión en común. No obstante en estos espacios se vivenciaron los traumas de salir del aislamiento. Los encuentros entre pares alternaron entre la marca del territorio propio de cada asignatura y la búsqueda de consensos. La marca de asignatura se impone, como exigencia en la determinación de conceptos, o procedimientos que unos profesores pretenden hacer prevalecer frente a otros, en el entendido de que dichos saberes forman parte del corpus de conocimientos propios de su asignatura. Este hecho genera la tensión, a la que muchos informantes refieren, aludiendo a la dificultad vivida en las salas generales para ponerse de acuerdo.

Obviamente no es sencillo especificar qué campo del saber tiene la autoridad intelectual a la hora de determinar qué es argumentar o qué es un informe, ya que necesariamente adquirirá distintas connotaciones, desde los géneros discursivos abordados por la lengua o desde los procedimientos protocolares establecidos por la física o la química.

De hecho, centrar la discusión en este punto es conducirla a un callejón sin salida, porque cada término adquirirá su precisión técnica en el ámbito propio de cada especialidad. 
A las dificultades de comunicación que interponen los tecnicismos propios de las jergas de asignatura, que como vimos cumplen el efecto secundario de otorgar una marca de materia y de delimitar un territorio propio de cada campo del saber, se suma el obstáculo muy sentido por el colectivo docente, de la falta de tiempo disponible para realizar tareas coordinadas desde una perspectiva multidisciplinaria.

Los nuevos espacios de encuentros requieren nuevos acuerdos de comunicación.

\section{Segundo Nivel de indagación. Una zona de antinomias}

\section{Contenidos versus competencias}

El segundo nivel de análisis atiende a las implicancias que para el estudiantado tiene la innovación curricular desde la mirada de los docentes. La preocupación más sobresaliente giró en torno a la creencia de que el trabajo tendiente al desarrollo de competencias, pone en peligro la adquisición de contenidos educativos. Se manifiesta también, un temor por la trivialización la enseñanza, masificándola en desmedro de la calidad. Esto suscitó sentimientos de pérdida, de ausencia de insumos teóricos, o de los tradicionales temarios que poblaron los cursos del bachillerato.

En lo particularmente concerniente a la evaluación, estas ideas vertebradoras giran en torno a los conceptos de desarrollo de competencias en detrimento de la repetición memorística de contenidos y una evaluación procesual que pone el acento en la totalidad del proceso de aprendizaje de los estudiantes y no solo del resultado valorado en el examen final.

Retomando la definición de Meirieu (2002) de competencias en tanto saberes identificados que ponen en juego varias capacidades dentro de un campo nocional o disciplinario, comprendemos que saber y saber hacer son dos caras de una misma moneda y de ninguna manera experiencias aislables, pues ciertamente ninguna capacidad puede expresarse en el vacío ni los saberes pueden manifestarse sin poner en juego ciertas capacidades.

\section{¿Sacar de foco el examen?}

La propuesta de sacar de foco al examen escandalizó a algunos profesores mientras que otros vivieron un proceso de desacralización de su figura del examen..

La batería de rutinas de evaluación, instituyó relaciones entre profesores y alumnos caracterizadas por la inautenticidad, el temor y el ocultamiento, ya que la calificación fue el centro sobre el que giró la actividad del profesor y la preocupación del alumno. Existe conciencia respecto a que la educación debe cimentarse en bienes culturales que se aparten de prácticas que giran en el vacío o conducentes a un aprendizaje frágil, en tanto mera transmisión de contenidos situados fuera de la vida y de los intereses de los estudiantes. No obstante se instala una zona de vacilación frente a procesos de aprendizaje que si bien denotan progresos significativos, no alcanzan los niveles de exigencia impuestos por el profesor como indicadores de logro.

\section{Los expertos ocultadores}

Paradójicamente las prácticas de evaluación centradas en el acierto del producto final, condujeron a un ocultamiento de los procesos de aprendizaje que dejan al desnudo el no saber. Por sobre todo, el estudiante debió demostrar que sabía. Desde una concepción de la evaluación de procesos, conocer las falencias y errores conceptuales de los estudiantes es crucial para reorientar la enseñanza. 
Tanto se castigó el error con la calificación, que los alumnos terminaron por ocultar sus dificultades convirtiéndose en expertos ocultadores. El mayor problema que a este respecto, enfrentan los profesores en una etapa incipiente del cambio, es no saber cómo llevar a cabo las reorientaciones de sus prácticas. Perciben el síntoma pero se les ocultan las causas. Tienen delante un alumno que redacta mal o lee mal, pero le cuesta saber exactamente en qué puntos radican dichas dificultades y naturalmente el estudiante no facilita el dato, por el contrario, procura ocultarlo.

La lógica de esta conducta radica en que exponer la ignorancia no lo ha ayudado hasta ahora, y es razonable que desconfíe de la ayuda que el profesor le ofrece. A esta dificultad intrínseca a la relación establecida entre el profesor y el alumno, se suman otras extrínsecas como el crecimiento de la matrícula y la existencia de aulas superpobladas las cuales entre sus efectos más perjudiciales tiene el de crear la dificultades de que el profesor conozca a sus alumnos y consecuentemente, la imposibilidad de atender la diversidad.

\section{Cuando preguntar puede jugar en su contra}

Como se expuso, es frecuente que el diálogo profesor- alumno en el aula, esté sesgado por la inautenticidad. Gran parte de las preguntas que el profesor realiza a sus alumnos, no son genuinas, sino que forman parte del andamiaje por él ideado, que lo conducen por terreno firme, al núcleo temático que desea presentar a sus alumnos.

Por su parte, los alumnos rara vez realizan preguntas genuinas, ya sea siguiendo la estrategia de camuflaje de lo ignorado, ya sea porque temen exponerse a la burla o al escarnio del grupo de clase, al preguntar lo que es obvio para otros, o sencillamente porque no obtienen respuesta.

Probablemente en una valoración costo / beneficio, dejar al descubierto la duda, tiene para el alumno, el riesgo de exponerse a la ironía, la burla y otras veces, lo que es aún más grave, dejar en evidencia que no sabe o está equivocado.

Obviamente el vínculo entre pares es menos riesgoso, por lo que es de esperar que las preguntas genuinas queden para después, para el recreo, para la casa, para la clase particular o para cualquier otra instancia de aprendizaje, en la que el estudiante se sienta a salvo de ser sorprendido en su impericia.

Otras veces la pregunta del alumno se descalifica, sencillamente porque cae fuera del corpus nocional que el profesor trazó para su curso, dentro de sus dominios, o hace referencia al territorio conceptual propio de un colega. La mención del alumno de la temática o la bibliografía recomendada por otro profesor, se percibe como una infiltración del otro, en un corpus disciplinar que el profesor ha adoptado como propio y que pretende preservar. Por tanto, vive esta circunstancia de manera muy próxima a la agresión.

\section{La Atención Flotante}

Aun en las situaciones en las que los diálogos que se establecen en la clase revisten un mayor grado de autenticidad, la relación que el profesor establece con el alumno parece no lograr atravesar el caparazón que lo aparta del mundo vivencial de los estudiantes.

Frecuentemente el profesor logra una gran adhesión de sus alumnos a sus propuestas, que siguen sus consignas, dejándose conducir por los andamios que él edificó para que ellos transiten. La clase incluso puede tener una dinámica signada por una participación muy nutrida.

No obstante, dada la falta absoluta de cuestionamientos por parte del alumno y ese ir y venir desde los asuntos de la clase a los asuntos privados, tan evidente en la alternancia de la mirada entre el pizarrón y el patio o la esquela que circula de mano en mano, dejan al aula transida de una artificialidad, que se podría traducir en una actitud básica de estar y no estar, en una suerte de atención flotante. 
De manera que la inautenticidad del encuentro educativo, no está necesariamente relacionada con la distancia de las propuestas escolares con la vida real de los estudiantes, sino con la falta de compromiso de la identidad de los seres sociales intervinientes, en la experiencia educativa.

Barbier,J. (1999) señala que enseñar no es trasmitir conocimientos, sino organizar experiencias educativas en las que está en juego la identidad de los sujetos participantes. En dicha identidad diferencia tres componentes: Ios de representación o saberes; los operativos, sintetizados en el saber-hacer y los afectivos que implican compromiso, gusto, disposición e interés por las propuestas de aprendizaje.

Las actividades del aprendizaje son movilizadoras de la identidad, -en tanto ponen en juego lo que se sabe de antes, lo que se quiere saber-, y productoras de la identidad en tanto generan el trabajo interactivo y comprometido con los otros.

La implicancia afectiva de lo que se hace en el universo escolar, en tanto valorización simbólica, de la utilidad de lo que se realiza y del reconocimiento social del entorno del que se forma parte, es sin duda un componente esencial en el aprendizaje genuino.

En este marco del quehacer comprometido con los otros, las actividades llevadas a cabo en el liceo, importan en sí mismas, más allá de las acreditaciones individuales. Los emprendimientos conjuntos entre enseñantes y aprendientes exponiendo sus identidades a la mirada de los demás, habilitan el placer colectivo de los logros y la tolerancia a los fracasos, en tanto todos se sienten hacedores y partícipes de las acciones ideadas y operativizadas en conjunto.

Desde esta perspectiva parece razonable que el estudiantado adopte una actitud distante, preservando su identidad frente a actividades que no parecen importar realmente a nadie ni siquiera al profesor que las propone, quien, con frecuencia parece más preocupado por dar cumplimiento al temario propuesto en cada unidad del programa, que en crear situaciones de enseñanza que movilicen al grupo intelectual y afectivamente.

José Esteve, en una entrevista realizada por Ciancio G. (2003) aborda una temática relacionada con la inautenticidad en el aula, al reflexionar acerca de la pseudo información y de la pseudo comunicación, presente en el mundo actual, especialmente a partir de los medios masivos de comunicación.

Tal vez en ninguna otra época como en la actual, los seres humanos, hayan estado tan expuestos a la comunicación ficticia que parece real, a partir de ciertos fenómenos masivos. Producciones mediáticas como los reality show, o los chat en internet crean una pseudo comunicación, en la cual absolutamente todo puede decirse, dejando fuera la identidad y consecuentemente el compromiso de quien dice, condición esencial de los actos de habla.

La atención flotante a la que se hace mención, como entidad observable en las aulas es un fenómeno complejo, enmarcado en un contexto histórico, donde la pseudo comunicación entre los humanos es una realidad recurrente, fuera y dentro de las instituciones educativas. Tal vez constituya, un mecanismo defensivo de quienes quieren preservar su identidad de lo repetitivo, lo trivial o lo intrusivo, en tanto contenidos prescriptos que pretenden imponerse sin negociación, ni compromiso afectivo e intelectual mediante. 
En definitiva en el aula reina una suerte de desaliento de quienes tienen poca esperanza de encontrar allí, algún desafío, algún reto al intelecto o a la emotividad, que ponga en marcha la energía y el esfuerzo imprescindibles para el aprendizaje y la producción creativa.

El traer al liceo al buen alumno y buen docente que cada uno quiere ser, -dejando en casa a la persona compleja que cada uno es, con sus certezas, con sus ignorancias, sus dudas, sus preguntas y sus respuestas a medias-, explica en gran medida esa falta de autenticidad del encuentro cotidiano.

\section{Tercer nivel de indagación: Las prácticas de enseñanza. Lo nuevo, lo viejo, lo amenazante}

El tercer y último nivel plantea las implicancias que el nuevo plan ha tenido en las prácticas de la enseñanza y en el reperfilamiento del rol docente. En este nivel se observaron esfuerzos muy interesantes por trascender a la posición primaria de repetición de contenidos, proyectándose trabajos que estimulan el desarrollo de habilidades complejas. Para ello se idearon estrategias intentando evitar el efecto de desconcierto que las nuevas prácticas de enseñanza suelen provocar en estudiantes habituados a propuestas tradicionales de formatos esclerosados, lo que supuso re pensar el oficio de enseñar.

\section{Construyendo una evaluación de procesos}

El hecho de que la innovación curricular ponga en el centro de la evaluación al proceso seguido por el alumno es bien valorado por el colectivo docente, aunque nadie parece ignorar el esfuerzo que supone construir una evaluación que atienda los procesos. Si los exámenes tradicionales se limitaron a constructos didácticos que solo exigían del estudiante un nivel de rescate de información, el desafío que enfrentan los profesores es generar instancias que permitan valorar el proceso de cada alumno, trascendiendo este nivel primario de repetición de contenidos, para proyectar trabajos que supongan un segundo nivel que implique el desarrollo de habilidades, como vincular temas, establecer comparaciones, aplicar conceptos y finalmente un tercer nivel de resolución de problemas que ponga en juego la creatividad.

En primer término comienza a gestarse la idea de que la evaluación de procesos viene necesariamente de la mano de la atención a la diversidad. La prueba diagnóstica instalada en las prácticas de evaluación de todos los Centros (en algunos, el docente debe adjuntarla al libro del profesor, en otros debe entregarla a la dirección con el correspondiente estudio estadístico y redacción de conclusiones), evidentemente se ha burocratizado en la medida que no es tenida en cuenta, como insumo primario, en el momento del diseño de las consignas de trabajo.

En segundo lugar, si la evaluación debe formar parte permanente del proceso ¿por qué concebirla como segmentos añadidos a modo de pruebas semestrales? Frente a este legítimo cuestionamiento, la percepción más generalizada apunta a que las pruebas como instrumentos formales contribuyen a desarrollar la responsabilidad del propio aprendizaje, en tanto no solo hay que aprender sino también acreditar.

Los profesores dejan al descubierto la inexistencia de verdades categóricas, ya que si bien, en la valoración del proceso de aprendizaje es más relevante el recorrido del estudiante, que el producto final, aun esto último depende de circunstancias contextuales. En consecuencia algunos entienden que en el momento de fijar los niveles de exigencias debe tenerse especialmente en cuenta el curso y la orientación. 


\section{Un arduo camino: La construcción de propuestas áulicas innovadoras}

Una de las características que ha asomado con mayor fuerza en el proceso de cambio que tiene lugar en el liceo de referencia es que, consignas innovadoras culminan, frecuentemente, con resoluciones muy tradicionales que llevan la actividad del aula al terreno en el que el profesor quiere situarla. Las artes de ocultación, a las que se hizo referencia, presentes en profesores y alumnos constituyen uno de los mayores obstáculos para vitalizar el potencial didáctico, presente en muchas de las propuestas de trabajo y consignas de pruebas e informes.

La inclusión de textos de diversa índole: gráficos, mapas, textos escritos, a los efectos de aplicar conceptos, inferir conclusiones, esgrimir argumentos o desarrollar otras capacidades, a partir de la decodificación de los mismos, son algunos de los instrumentos más empleados por los profesores que intentan introducir cambios en sus propuestas didácticas. La sustitución de los tradicionales ejercicios por las propuestas de resolución de problemas, es otra de las características observadas, fundamentalmente, en el área de las ciencias.

Procurando establecer una tipología de las pruebas propuestas por los docentes, las mismas podrían clasificarse en:

- Pruebas abstractas sin anclaje en la realidad.

- Repetitivas de formatos esclerosados

- Pruebas que suponen un reto justo.

- Pruebas complejas, tendientes a la resolución de problemas.

Cabe aclarar que esta taxonomía solo es válida en las condiciones y características que reviste el caso estudiado. Recordemos que se trata de un liceo de bachillerato que está transitando una innovación curricular y los profesores están adecuando sus instrumentos de evaluación a los nuevos requerimientos, por lo cual es de esperarse, que lo que hoy se considere una prueba compleja, mañana probablemente no lo sea. Lo cierto es, que en las actuales condiciones, el pasaje de pruebas sedimentadas por la tradición hacia la formulación de nuevas propuestas más afines con el espíritu de la innovación curricular, es un proceso que reviste características muy diversas y que merece ser analizado.

En esta transición como ya se mencionara, el profesor se debate entre lo repetitivo, lo trivial y por otro lado, lo innovador que con frecuencia desconcierta al estudiante, que aún no ha detectado con claridad, los indicios del cambio, dejándose sorprender, mal situado, por propuestas pedagógicas para las que no está preparado.

Las pruebas incluidas en la categoría de reto justo son generalmente aquellas que evitan la mera repetición, procurando establecer situaciones-problema vinculadas a la vida del alumno, o contextualmente situadas y que a su vez contemplan distintos niveles de complejidad. De esta manera se busca que cada estudiante, pueda transitar por ellas, hasta el nivel al que logre acceder. Estas pruebas en general incluyen algunas preguntas de rescate de información o de ejercitación mecánica para evitar la frustración de quienes no están preparados para resolver situaciones problemáticas.

Para evitar el efecto desconcierto al que se hizo referencia, frente a las propuestas no tradicionales, las estrategias más empleadas por los docentes son:

- Propuestas de resolución de problemas en equipos.

- Pruebas que incluyen distintos niveles de complejidad, procurando evitar la descompensación emotiva o la frustración al enfrentar formatos no convencionales. 
El análisis de documentos de propuestas de pruebas, permite conocer los caminos por los que los profesores han venido transitando en este arduo proceso de ir ubicándose frente a los cambios.

La mayoría de los constructos didácticos elaborados, revelan las huellas de los acuerdos de sala docente aun cuando no siempre se logre sobrepasar el nivel de pruebas repetitivas. En algunas de ellas prevalece el esfuerzo por acercarse al mundo vivencial de los estudiantes con textos que consideran pertinentes; otras ponen el acento en el desarrollo de competencias y la especificación clara de lo que se espera del alumno, haciendo constar puntos y habilidades que deben ponerse en juego, mientras otras apuntan a alcanzar el nivel de resolución de problemas con implicancia de operaciones lógicas y creativas.

Entre estas últimas es muy interesante la estrategia empleada por varios profesores que intercalan actividades tradicionales de ejercitación o repetición de conceptos con situaciones complejas que incluyen la resolución de problemas, como manera de enfrentar la diversidad, respetando los distintos niveles del desarrollo del aprendizaje de sus alumnos.

La vida cotidiana del aula deja de manifiesto esfuerzos muy notorios por innovar, que se evidenciaron en una etapa incipiente en el proceso de transformar las prácticas de evaluación, y que suponen grandes desafíos para los colectivos docentes:

\section{Algunas reflexiones en torno políticas educativas actuales.}

El camino recorrido, permite valorar las innovaciones curriculares referidas al sistema de evaluación, implementadas por la ANEP (Administración Nacional de Educación Pública) como una etapa incipiente en el proceso de transformar las prácticas de evaluación, con el objeto de posibilitar un menor rezago, una mayor inclusión y una efectiva retención educativa.

Podrían considerarse dos grandes desafíos a nivel de las políticas educativas:

En primer término habilitar en la totalidad de las matrices de los planes educativos, espacios curriculares que permitan la realización de salas docentes, salas de coordinación, creación de equipos institucionales, con el objeto de contribuir a la diversificación del rol docente especialmente en lo atinente a un mayor protagonismo en áreas educativas que trasciendan al aula. Respecto a estos espacios curriculares, se evidencia, la necesidad de implementarlos y construirlos para convertirlos en lugares genuinamente productivos. Las instituciones deberán enfrentar las tensiones que generan las marcas de asignaturas, propias de colectivos docentes que han limitado su accionar a la esfera del aula y su campo interactivo a coordinaciones entre colegas de la misma asignatura.

Ampliar las esferas de interacción, potenciando la participación de los actores sociales en los proyectos institucionales, procesos de seguimiento y autoevaluación de los centros educativos diseños curriculares y producción de conocimiento, son puntos medulares en los que los diferentes niveles de la gestión pública tendrán un rol preponderante. A este respecto, la presente investigación evidenció la relevancia del reconocimiento social que las instituciones educativas realizan a la labor de sus integrantes, con relación al crecimiento de la autoestima, la autonomía y el desarrollo de la profesionalización de quienes sienten que sus voces son escuchadas y su trabajo valorado. 
En segundo término, respecto de los sistemas de evaluación que contemplan instancias de corte como pruebas semestrales y trabajos interdisciplinarios para facilitar una evaluación de procesos, se entiende que sin bien estos instrumentos han sido, en primera instancia, legítimamente cuestionados por docentes que temen abandonar la cultura del examen fuertemente arraigada en educación media, los procesos interactivos fueron progresivamente, dando lugar a la construcción de los nuevos dispositivos de evaluación, emergiendo una percepción predominantemente positiva acerca de los mismos.

A nivel de los colectivos docentes los desafíos actuales suponen:

En primer lugar, rescatarse mutuamente del aislamiento, revitalizando al docente como productor de conocimiento, en este complejo y apasionante arte de enseñar. Para ello será imprescindible potenciar y consolidar los espacios curriculares ganados.

En segundo lugar, serán fundamentales los esfuerzos por romper el círculo de ocultamiento e inautenticidad reinante en el aula, propiciando relaciones más humanas, en las que preguntar no sea un riesgo y aprender del error sea válido y deseable. Relaciones en las cuales el profesor busque y propicie los desafíos de sus alumnos y donde el mayor compromiso ético que pueda asumir con ellos, no sea saber sino transitar juntos, en la búsqueda del conocimiento, adentrándose en la espesura de lo ignorado para salir reconfortados y vitalizados por el placer del descubrimiento.

\section{Bibliografía.}

ALVAREZ MENDEZ, J. 2000. Didáctica Currículo y evaluación. Ensayos sobre cuestiones didácticas. 2da.ed. Madrid: Niño y Dárila Editores.

BARBIER J. M. 1993. La evaluación en los procesos de formación. Temas de Educación. Buenos. Aires: Paidós.

BARBIER, J. M. 1999. Prácticas de formación. Evaluación y análisis. Buenos Aires: Ediciones de la Facultad de Filosofía y Letras. UBA. Novedades Educativas.

BRUNER, J. 1997. La educación Puerta de la Cultura. Madrid: Visor.

BRUNER, J. 1997. La Fábrica de historias, derecho, literatura, vida. Buenos Aires: Fondo de Cultura económica de Argentina.

CARBONELL, J. 2001 La aventura de innovar. El cambio en la escuela. Madrid: Morata.

CAREAGA, A. 2003. Una aproximación a la evaluación práctica docente desde una perspectiva colaborativa. Montevideo: AECI.

CIANCIO, G. 2003 Cambian los escenarios, cambian los educadores: una conversación con José Esteve. En : Educar 6(13): pp. 22-28.

COMISIÓN TEMS / ANEP. 2003. El Cambio Curricular En : Cuadernos de trabajo. Serie Aportes para la Reflexión y la Transformación de la EMS. 1(17) pp: 7-55.

COMISIÓN TEMS / ANEP. 2003. Jornada escolar, estudio y exámenes en el Bachillerato Secundario. En: Cuadernos de trabajo. Serie Aportes para la Reflexión y la Transformación de la EMS. 1(20) pp: 7-26. 
CONTRERAS, J. 1997. La autonomía del profesorado. Madrid: Morata.

DAVINI, M. 1997. La Formación docente en cuestión: política y pedagógica. Buenos Aires: Paidós.

DÍAZ BARRIGA, A. 1992 Curriculum y evaluación escolar. Buenos Aires: Aiqué.

DÍAZ BARRIGA, A. 1994. Docente y programa. 2da. ed. Buenos Aires: Aiqué.

EISNER, E. 1998. El ojo ilustrado. Indagación cualitativa y mejora de la práctica educativa. Barcelona: Paidós.

EISNER, E. 2002. La escuela que necesitamos. Buenos. Aires: Amorrortu.

ELLIOT, J. 2000. El cambio Educativo desde la Investigación Acción. 3era. ed. Madrid: Morata. FENSTERMACHER, G. Tres aspectos de la Filosofía de la Investigación sobre la enseñanza. En: WITTROCK, M.1989. La Investigación de la Enseñanza. Tomo I. Barcelona: Paidós.

FULLAN, M.; HARGREAVES, A. 1996. La Escuela que queremos. Buenos Aires: Amorrortu. GOODSON, I. 2003. Estudio del Currículum. Casos y Métodos. Buenos Aires: Amorrortu.

JACKSON, P. 1968. La vida en las Aulas. Madrid: Morata.

JAKSON, P. 1999. Enseñanzas Implícitas. Buenos Aires: Amorrortu.

LISTON, D.; ZEICHNER, K. 1997. Formación del Profesorado y Condiciones Sociales de la Escolarización. 2da. ed. Madrid: Morata.

LITWIN, E. 1997. Las Configuraciones didácticas. Buenos Aires: Paidós.

LOPATER, A. et al. 2003. Claves para entender el Nuevo Reglamento de Evaluación y Acreditación de carácter experimental para la educación media Superior, Modalidad General. En: Boletín temático Infotems. 1 (1) pp:4-8.

MCEWAN, H.; EGAN, K .1998. La narrativa en la enseñanza, el aprendizaje y la investigación. Buenos Aires: Amorrortu.

MEIRIEU, P. 2002. Aprender sí pero ¿cómo? 3era. ed. Barcelona: Octaedro.

NUÑEZ, V. ¿Qué se quiere decir con evaluar? En: TIZO,H. 2003. Reiventar el vinculo Educativo: aportaciones de la pedagogía Social y del Psicoanálisis. Barcelona: Gedisa.

PARDINAS, F. 1998. Metodología y Técnicas de investigación en Ciencias Sociales, 35ta. ed. Madrid: Siglo veintiuno.

PERKINS, D. 2001. La escuela Inteligente. 2da. ed. Barcelona: Gedisa.

PERRENOUD, P. 1996. La construcción del éxito y del fracaso escolar. 2da.ed.Madrid : Morata.

RODRIGO, M., et al. 1993. Las Teorías Implícitas. Madrid: Visor.

SANTOS GUERRA, M. 1996. Evaluación Educativa. Buenos Aires: Ed. Magisterio del Río de la Plata. 
SANTOS, M A. 1993. Hacer visible lo Cotidiano. 2da. ed. Teoría Práctica de la Evaluación Cualitativa de los Centros escolares. Madrid : Akal.

SARASON,S. 2002. La enseñanza como arte de representación. Buenos Aires: Amorrortu. SAUTU R. 2003. Todo es teoría. Buenos. Aires: Ediciones Lumiere.

TAYLOR,S.J.; BOGDAN,R.1987. Introducción a los métodos cualitativos de investigación. Barcelona: Paidós.

TORP, L.; SAGES, S. 1999. El aprendizaje basado en problemas. Buenos Aires: Amorrortu. VALLES, M. 1999. Técnicas cualitativas de investigación Social. Madrid: Ed. Síntesis S.A.

*El Artículo se enmarca en la tesis de maestría de la autora, dirigida por el Dr. Daniel Germán. Año 2005.

**Master en Educación, Universidad ORT. Licenciada en Servicio Social. Profesora de Literatura. Docente en Institutos de Formación Docente, en Investigación Educativa. 